\title{
Análisis de reducción de dimensiones para el reconocimiento de actividades físicas humanas usando fusión multimodal
}

\author{
Dimension reduction analysis for the recognition of human \\ physical activities using multimodal fusion
}

\author{
Daniel Rengifo Almanza ${ }^{1} \quad$ Andrés Calvo Salcedo $^{1} \quad$ Carlos Henao Baena $^{2}$ \\ Recibido 1 de septiembre de 2020, aceptado 7 de octubre de 2021 \\ Received: September 1, $2020 \quad$ Accepted: October 7, 2021
}

\begin{abstract}
RESUMEN
El reconocimiento automático de actividades físicas humanas es una tarea importante en aplicaciones de visión por computador. Los enfoques robustos que utilizan unos o varios sensores generalmente vinculan características redundantes que consumen recursos computaciones y tiempo de cómputo durante el proceso de clasificación de subactividades. En este artículo se explora la reducción de dimensión para el reconocimiento de actividades y movimientos primitivos humanos fusionando datos provenientes de sensores de profundidad visual Kinect, sensores inerciales IMUs y electrodos de registro electromiográfico (EMGs). También se enseña un estudio comparativo donde se evalúa diferentes técnicas de reducción de características del estado arte, se analiza el comportamiento en base al desempeño en el reconocimiento de actividades física y el tiempo de cómputo de los métodos bajo estudio. Los resultados muestran que, metodologías del estado de arte pueden tener un menor costo temporal a la hora de su implementación sin afectar considerablemente el desempeño al reconocer la actividad.
\end{abstract}

Palabra clave: Actividades físicas, movimientos primitivos, reducción de dimensiones, aprendizaje de máquina.

\begin{abstract}
Automatic recognition of human activities is an important task in computer vision applications. Robust approaches using one or more sensors generally link redundant features that consume computing resources and computation time during the sub-activity classification process. This article explores dimension reduction for recognizing human activities and primitive movements by merging data from Kinect visual depth sensors, IMUs inertial sensors, and electrodes of electromyographic record (EMGs). It shows a comparative study where the different techniques of reduction of state of the art are evaluated characteristics; behavior is analyzed based on performance in recognition of activities and computation time of the methods under study. The results show that state-of-the-art methodologies could have a lower temporary cost when implementing them without significantly affecting performance when recognizing the activity.
\end{abstract}

Keywords: Physical activities, primitive movements, dimensions reduction, machine learning.

1 Universidad Tecnológica de Pereira. Facultad de Ingenierías. Perera, Colombia.

E-mail: dfrengifo@utp.edu.co; afcalvo@utp.edu.co

2 Gestor Línea de Electrónica y Telecomunicaciones Tecnoparque Nodo Pereira, Centro Atención Sector Agropecuario - SENA. Pereira, Colombia. E-mail: c_henao_86@hotmail.com

* Autor de correspondencia: Carlos Alberto Henao: c_henao_86@ hotmail.com 


\section{INTRODUCCIÓN}

El estudio de actividad física humana es una temática de interés debido a las aplicaciones que requieren de este tipo de análisis. Algunos ejemplos son la rehabilitación de pacientes, cuidado de enfermos, seguridad, monitoreo y seguimiento de elementos móviles. Esta tarea ha sido constituida a través de sistemas expertos que utilizan sensores electrónicos para la caracterización e identificación de los movimientos $[1,2,3,4,5]$.

Para la implementación, se requieren sensores de movimiento y técnicas modernas de análisis de datos. Algunas de las variaciones de sensor más comunes en esta tarea son: Cámaras de profundidad, sensores de masa inercial (IMU) y electromiográficos (EMG). Aunque se han realizado diferentes estudios con información proveniente de estas modalidades de sensor, cada enfoque ha demostrado tener ventajas y desventajas ante diferentes escenarios $[1,4,6,7]$.

En la investigación [1] se plantea una metodología basada en movimientos primitivos para la detección automática de actividades físicas, si bien en este se conservan elementos presentados en $[6,8]$, este trabajo introduce el uso de la fusión de sensores (Kinect, IMU y EMG). Esta variante aumenta el desempeño en la clasificación de actividad aprovechando las virtudes de cada sensor, además de ofrecer alternativas de funcionamiento al sistema ante posibles fallas tales como auto oclusiones, desconexiones de los sensores y daños en los mismos [1]. Otro aporte importante de este trabajo es el cambio de un diccionario de posturas, por uno de micro-movimientos (movimientos primitivos), tal y como se plantea en el trabajo [6], permitiendo la asignación de etiquetas, no solo para el sensor Kinect, si no para las otras modalidades de sensores donde no existe la forma de asignar una etiqueta a las posturas para diferentes frecuencias de muestreo de los sensores. Sin embargo, este enfoque presenta un costo computacional alto, debido a que requiere de una SVM por modalidad de sensor para clasificar múltiples movimientos primitivos, lo que restringe su implementación en un sistema de respuesta en tiempo real. Por otro lado, en la etapa de extracción de características se reporta un alto costo computacional, debido a dos procesos, el primero es la cantidad de operaciones de cómputo que se requiere en la etapa de extracción de características y el segundo es la cantidad de múltiples fuentes sensoriales que se utilizan para la recopilación de los datos.

Específicamente la extracción de características es una de las etapas que más costo computacional requiere en una aplicación de aprendizaje de máquinas $[9,10]$. El problema se debe a que, en muchas situaciones, los datos tienen un gran número de variables o dimensiones que aumenta la cantidad y complejidad de procesos y cálculos. Por tanto, es imprescindible visualizar y analizar previamente el conjunto de datos de alta dimensión y así determinar las características que son relevantes para el reconocimiento de la actividad. En el estado del arte se ha propuesto técnicas para reducción de dimensionalidad y análisis de datos como lo son Análisis de Componentes PrincipalesPCA, la extensión de PCA- KPCA y t-SNE) [7]. Estas proveen herramientas para visualizar y calcular el aporte de los datos en espacios de dos dimensiones conservando en mayor parte la estructura que tienen en una alta dimensionalidad. Los métodos tradicionales de reducción tales como PCA, en el trabajo [11] y el escalado multidimensional clásico (MDS) son técnicas lineales de separación y algunos casos no representan las variaciones no lineales de los datos, generando un espacio de representación traslapando [12]. Para corregir el problema anterior, se han propuesto un gran número de técnicas de reducción no lineal que pretenden preservar la estructura local y global de los datos como lo son: Stochastic Neighbor Embedding (SNE), Isomap, Maximum Variance Unfolding (MVU), Locally Linear Embedding (LLE), and Laplacian Eigenmaps [12]. Estas han demostrado resultados satisfactorios en diferentes aplicaciones, sin embargo, se evidencia que dependen ampliamente de la naturaleza de los datos bajo análisis.

En este trabajo se realiza un estudio comparativo para la clasificación de actividades humanas aplicando diferentes técnicas de reducción de dimensionalidad reportadas en el estado del arte conservando la metodología propuesta en la literatura, además en el estudio que se utilizan técnicas de visualización en dos dimensiones para analizar la naturaleza de los datos. Por otro lado, se logra obtener resultados competitivos en el proceso de clasificación de la actividad física y reducción del costo computacional, así como en el número de características necesarias para construir la matriz de entrenamiento en la etapa de clasificación de micro movimientos. También es 
importante resaltar que se logra evidenciar un método de fusión de sensores con costo computacional menor al obtenido por metodologías enseñadas en la literatura. En síntesis, las principales contribuciones de este artículo son:

- Documentación de una metodología que permite reconocer actividades físicas humanas a partir de técnicas de análisis de dimensionalidad y aprendizaje supervisado.

- Reporte y evaluación de la metodología en función de diferentes técnicas de análisis de dimensionalidad del estado del arte.

Este artículo está organizado como sigue:

- En la Tabla 1 se muestran las definiciones de las siglas usadas en el artículos y que son poco conocidas.

- En la sección 2 la metodología es introducida, describiendo cada una de las etapas que la conforman, como los descriptores utilizados y métricas de análisis de resultados

- En la sección 3 se describen los resultados debatiendo las ventajas, los beneficios y los inconvenientes encontrados, por último, las conclusiones de la investigación son presentadas.

\section{NOMENCLATURA PARA EL TRABAJO}

\section{TRABAJO PREVIO}

Para detección de actividades algunos estudios han propuesto utilizar técnicas de visión por computador, sin embargo, estos se ven afectados por las condiciones fotométricas de la escena y así como por el ruido introducido por el sensor de medida [13]. Buscando soluciones más robustas, algunas investigaciones proponen uso del sensor 3D de Microsoft Kinect para el reconocimiento de la actividad física basado en métodos de aprendizaje de máquinas $[2,14]$. Se utiliza clasificadores no supervisados (K-means, KNN, Spectral Clustering) para generar un libro de códigos con posturas clave, posteriormente emplear un Modelo Oculto de Markov (HMM) para reconocer las diferentes combinaciones de posturas e identificar así la actividad que se está realizando [6-8]. Un método que aplica una técnica similar y logra resultados importantes, consiste en utilizar una Máquina de Vectores de Soporte (SVM) para la codificación del libro de códigos, que para efectos de este trabajo se conocen como movimientos primitivos; luego con la HMM se reconoce las secuencias generadas por la ejecución de la actividad [8]. En contraste de otros enfoques, la SVM logra desempeños mayores a las técnicas no supervisadas, si bien, los métodos que

Tabla 1. Definiciones.

\begin{tabular}{|l|l|}
\hline \multicolumn{1}{|c|}{ Nomenclatura } & \multicolumn{1}{c|}{ Descripción } \\
\hline$K I T F$ & Conjunto de Atributos totales Kinect \\
\hline$I M U F$ & Conjunto de atributos totales IMU \\
\hline$E M G F$ & Conjunto de atributos totales EMG \\
\hline$\Psi=x 1 \ldots . . x l$ & Vector de coordenadas cartesianas de puntos articulados Kinect \\
\hline$x l=X l Y l Z l$ & Coordenada espacial del sensor articulado $l$ \\
\hline$I k=a x$ ay az ar $a \theta$ & Vector de aceleración entregado por el sensor de movimiento $k$ \\
\hline$\rho \boldsymbol{l}=r \boldsymbol{l} \theta \boldsymbol{l}$ & Coordenadas polares del sensor articulado $l$ con respecto al centro de masa de $\Psi$ \\
\hline$r \boldsymbol{l}$ & Componente radial de $\rho l$ \\
\hline$\theta \boldsymbol{l}$ & Componente angular de $\rho l$ \\
\hline$E K$ & Vector de etiquetas Movimientos primitivos Kinect - SVM \\
\hline$E I$ & Vector de etiquetas Movimientos primitivos IMU - SVM \\
\hline$E E$ & Vector de etiquetas Movimientos primitivos EMG - SVM \\
\hline$E F$ & Vector de etiquetas concatenadas de movimientos primitivos Kinect - EMG - IMU \\
\hline$C P$ & Centro de masa de $\Psi$ \\
\hline$K I T F(a)$ & Conjunto de Atributos Kinect reducido \\
\hline$I M U F(a)$ & Conjunto de atributos IMU reducido \\
\hline$E M G F(a)$ & Conjunto de atributos EMG reducido \\
\hline $\mathrm{N}$ & Tamaño del vector de magnitud de la FFT \\
\hline
\end{tabular}


Tabla 2. Descriptores.

\begin{tabular}{|c|c|}
\hline Definición & Descripción \\
\hline $\begin{array}{l}\text { Media de } X_{l}, Y_{l}, Z_{l} \text { normalizada al centro } \\
\text { de masa de } \Psi\end{array}$ & $m_{x}=\frac{1}{3} \sum_{i=1}^{3}\left(X_{i}-C P\right) ; m_{y}=\frac{1}{3} \sum_{i=1}^{3}\left(Y_{i}-C P\right) ; m_{z}=\frac{1}{3} \sum_{i=1}^{3}\left(Z_{i}-C P\right)$ \\
\hline $\begin{array}{l}\text { Media de } r_{l}, \theta_{l} \text { normalizada al centro } \\
\text { de masa de } \Psi\end{array}$ & $m r=\frac{1}{3} \sum_{i=1}^{3} r_{i} ; m_{\theta}=\frac{1}{3} \theta_{i}$ \\
\hline Varianza de $X_{l}, Y_{l}, Z_{l}$ & $\begin{array}{l}v_{x}=\frac{1}{3} \sum_{i=1}^{3}\left(X_{i}-C P-m_{x}\right)^{2} \\
v_{y}=\frac{1}{3} \sum_{i=1}^{3}\left(Y_{i}-C P-m_{y}\right)^{2} \\
v_{z}=\frac{1}{3} \sum_{i=1}^{3}\left(Z_{i}-C P-m_{z}\right)^{2}\end{array}$ \\
\hline Varianza de $r_{l}, \theta_{l}$ & $v_{r}=\frac{1}{3} \sum_{i=1}^{3}\left(r_{i}-m_{r}\right)^{2} ; v_{x}=\frac{1}{3} \sum_{i=1}^{3}\left(\theta_{i}-m_{\theta}\right)^{2}$ \\
\hline Medida de $a_{x}, a_{y}, a_{z}, a_{r}, a_{\theta}$ & $\begin{array}{c}m_{a x}=\frac{1}{3} \sum_{i=1}^{3} a_{x i} ; m_{a y}=\frac{1}{3} \sum_{i=1}^{3} a_{y i} ; m_{a z}=\frac{1}{3} \sum_{i=1}^{3} a_{z i} \\
m_{a r}=\frac{1}{3} \sum_{i=1}^{3} a_{r i} ; m_{a \theta}=\frac{1}{3} \sum_{i=1}^{3} a_{\theta i}\end{array}$ \\
\hline Varianza de $a_{x}, a_{y}, a_{z}, a_{r}, a_{\theta}$ & $\begin{array}{l}v_{a x}=\frac{1}{3} \sum_{i=1}^{3}\left(a_{x i}-m_{a x}\right)^{2} ; \\
v_{a y}=\frac{1}{3} \sum_{i=1}^{3}\left(a_{y i}-m_{a y}\right)^{2} ; \\
v_{a z}=\frac{1}{3} \sum_{i=1}^{3}\left(a_{r i}-m_{a z}\right)^{2} ; \\
v_{a r}=\frac{1}{3} \sum_{i=1}^{3}\left(a_{r i}-m_{a r}\right)^{2} ; \\
v_{a \theta}=\frac{1}{3} \sum_{i=1}^{3}\left(a_{\theta i}-m_{a \theta}\right)^{2} ;\end{array}$ \\
\hline Media de la intensidad del movimiento $A I$ & $A I=\frac{1}{3} \sum_{i=1}^{3} M I_{k}^{i} ; M I_{k}=\left\|{ }^{3}\left[\begin{array}{lll}\text { ax } & \text { ay } & a z\end{array}\right]\right\|$ \\
\hline Varianza de $A I(V I)$ & $V I=\frac{1}{3} \sum_{I=1}^{3}\left(M I_{k}^{i}-A I\right)$ \\
\hline $\begin{array}{l}\text { Área de magnitud de señal normalizada } \\
\text { SMA }\end{array}$ & $S M A=\frac{1}{3}\left(\sum_{I=1}^{3}\left|a x_{k}^{i}\right|+\left|a_{k}^{i}\right|+\left|a_{z k}^{i}\right|\right)$ \\
\hline $\begin{array}{l}\text { Valores propios de direcciones dominantes } \\
\text { EV A }\end{array}$ & $\begin{array}{l}\text { Vectores propios de la matriz de covarianza de la aceleración a lo largo } \\
\text { de los ejes } \mathrm{x}, \mathrm{y}, \mathrm{z} \text { en cada ventana de observación. }\end{array}$ \\
\hline \multirow[t]{2}{*}{ Energía de aceleración promedia $A A E$} & $\begin{array}{l}\text { Valor medio de la energía a lo largo de tres ejes de aceleración. Donde } i \\
\text { son las aceleraciones } a_{x}, a_{y} \text { y } a_{z \cdot} \\
\qquad A A E=\frac{1}{3} \sum_{i=1}^{3} \frac{1}{N} \sum_{j=1}^{N}\left|F F T\left\{a_{i}\right\}\right|^{2}\end{array}$ \\
\hline & $\begin{array}{l}\text { Donde el termino } F F T\{a i\} \text { corresponde a los coeficientes en el dominio } \\
\text { de la transformada rápida de Fourier de las aceleraciones }\left(a_{x}, a_{y} \text { y } a_{z}\right) \text {. }\end{array}$ \\
\hline Energía de rotación promedio & $\begin{array}{l}\text { Valor medio de la energía a lo largo de los ángulos de rotación, para este } \\
\text { caso solo } a_{r} \text { y } a_{\theta} \text {. Donde } i \text { son las aceleraciones } a_{r} \text { y } a_{\theta} \text {. } \\
\qquad A R E=\frac{1}{2} \sum_{i=1}^{2} \frac{1}{2} \sum_{j=1}^{N}\left|F F T\left\{a_{i}\right\}\right|^{2}\end{array}$ \\
\hline
\end{tabular}


utilizan videocámaras o cámaras de profundidad demuestran resultados confiables, estos presentan limitaciones causadas por oclusiones parciales del objetivo $[15,16]$.

Las unidades de medida inercial (IMU) también se utilizan para el reconocimiento de la actividad física, dando fuertes ventajas ante oclusiones de los objetivos analizados $[3,17]$. Por ejemplo, estos dispositivos (IMU) combinados con un modelo de detección basado en movimientos primitivos, logran generar un libro de códigos que realizan la detección de actividad con una eficiencia mayor al $90 \%$ [4]. Adicionalmente es posible perfeccionar el método anterior agregando el modelo de bolsa de características (BOF) con histogramas de símbolos primitivos, así se logra construir un conjunto de características que representan cada movimiento, logrando así obtener eficiencias del 92\% [4]. Aun cuando se logran resultados confiables, esta modalidad de sensor requiere altas capacidades de procesamiento [18], y en gran parte de los casos es necesario más de un sensor para realizar una detección satisfactoria de la actividad [1, 19, 17, 7].

Se evidencia que una sola modalidad de sensor generalmente es incapaz de identificar una amplia gama de actividades humanas [1], por lo que algunos enfoques proponen la fusión de datos de múltiples o diferentes sensores para mejorar el rendimiento de los sistemas de reconocimiento y así extender la cantidad de actividades a reconocer [19, 20]. Por otro lado, se destaca que se documenta diversos métodos para el reconocimiento con fusión multimodal [4, 3], sin embargo, pocos estudios analizan la unión de dos más modalidades de sensor.
Otros sensores que se utilizan en esta tarea son los de señales electromiográficas (EMG), los cuales permiten detectar las flexiones y contracciones de los músculos [3,21]. En la extracción de características de las señales EMG se han usado gran cantidad de metodologías que se pueden dividir en tres grandes grupos [17]. El primero es el enfoque estadístico, el segundo es un enfoque temporal y en tercer lugar, descomposición en frecuencia o espectral. En la primera clase se puede destacar el valor absoluto promedio (MAV) [1], cálculo de la entropía [3, 22, 23]. Dentro de los temporales se destaca el valor RMS [24, 15], y el análisis de variaciones de la señal (derivadas de primer y segundo orden) [20]. Dentro de enfoque espectral se podría encontrar técnicas como MFCC (Mel Frequency Cepstral Coefficient) [25, 26], Modelos Auto Regresivos (AR) [1, 3, 27], descomposición modal empírica [28] y la Transformada de Fourier y sus variaciones $[26,5]$. Aunque estas técnicas demuestran buenos resultados como descriptores, el procesamiento de las señales es complejo y requiere de múltiples sensores para lograr una detección competitiva $[4$, $18,7,29]$.

\section{MATERIALES Y MÉTODOS}

En la Figura 1 se enseña de forma general la propuesta de trabajo. En síntesis, esta se divide en cinco procesos principales. En primer lugar, la base de datos anotada, en segundo lugar, el procedimiento de extracción de características, luego en la tercera parte, el análisis de dimensionalidad, en el cuarto lugar clasificación de movimientos primitivos, por último, clasificación de actividades. A continuación, se describe cada uno de los procesos.

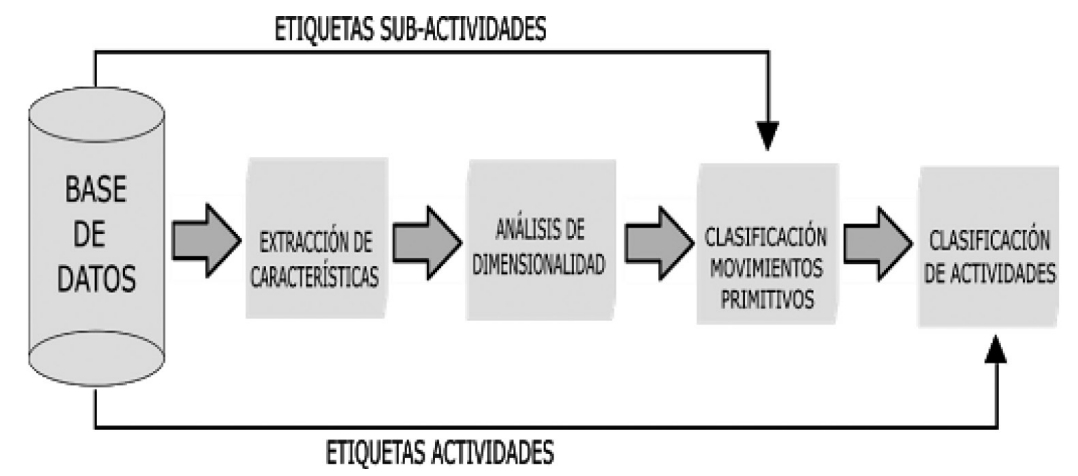

Figura 1. Metodología Trabajo. 


\section{BASE DE DATOS}

La colección de datos utilizados en este trabajo fue la suministrada por [1], esta se resume en la Figura 2, la cual fue construida con tres tipos de sensores (Kinect, IMU y EMG). La información fue etiquetada de acuerdo con cinco actividades principales. Por otro lado, como la ejecución de las actividades consta de una secuencia de acciones las cuales pueden ser divididas en ventanas de tiempo permitiendo descomponer cada una de estas en subactividades (movimientos primitivos). De esta manera los datos se etiquetan de acuerdo con las actividades y a cada uno de los sub-movimientos. Las salidas de la base de datos corresponden a los vectores $\psi, I k$ y $\boldsymbol{E} \boldsymbol{M} \boldsymbol{G}$.

\section{Extracción de características}

En esta etapa se computa un conjunto de descriptores de los datos. Estos representan las características que suministran cada uno de los sensores vinculados a la base de datos. Es de aclarar que se discrimina el cálculo de acuerdo con el tipo de instrumento. En el caso del Kinect el procedimiento es el siguiente, dado el vector de coordenadas cartesianas de puntos articulados $\psi$ se obtiene un arreglo de características $\rho i$ transformado cada articulación que pertenece a $\psi$ a coordenadas polares utilizando como referencia el centro de masa de $\psi$, acá $i$ representa el número de muestras de $\rho$. Luego se obtiene el conjunto de atributos $\boldsymbol{K} \boldsymbol{I T}$ dado por (ver ecuación 1):

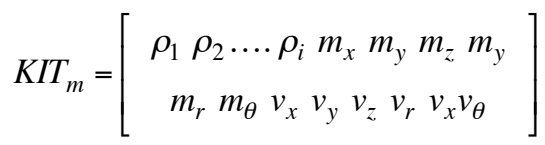

Donde, $m$ corresponde al $m$ - ésimo punto articulado del Kinect, de manera que para el conjunto de $m$ $\in 1 \ldots \ldots m$ se computa el vector $\boldsymbol{K I T F}$ como en el enlace de todos los KITm. Similarmente los sensores IMU entregan un vector de aceleraciones $I k$ donde $k$ es el k-ésimo sensor $(k \in 1 \ldots \ldots k)$, de esta forma dado el $I k$ se procede a encontrar los parámetros físicos del movimiento humano, así como los momentos estadísticos [1], obteniendo IMU $\boldsymbol{k}$ para cada sensor $k$ dado por (ver ecuación 2):

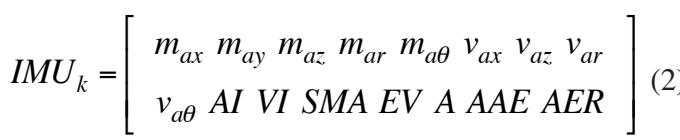

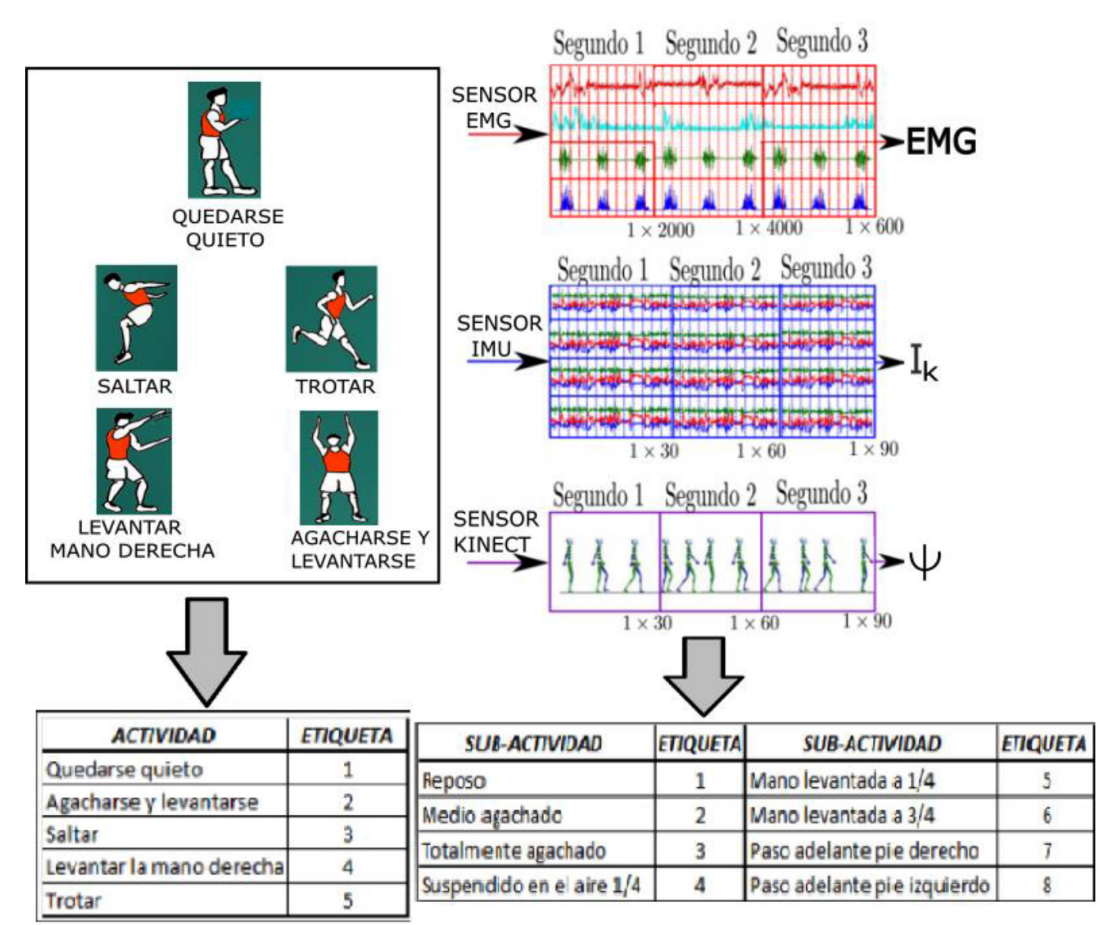

Figura 2. Estructura Base de datos. 
Así para el conjunto $k \in 1 \ldots \ldots k$ se tiene el vector $\boldsymbol{I} \boldsymbol{M} \boldsymbol{U} \boldsymbol{F}$ definido como la concatenación de $\boldsymbol{I} \boldsymbol{M U} \boldsymbol{k}$, esto es (ver ecuación 3):

$$
I M U F=\left[I M U_{1} I M U_{2} \ldots \ldots I M U_{k}\right]
$$

Para los EMG, se captura la actividad de cuatro músculos del cuerpo (ver Figura 3). La frecuencia de muestreo de cada canal en la captura es de $2 \mathrm{kHz}$, debido a que el sistema de adquisición de datos es

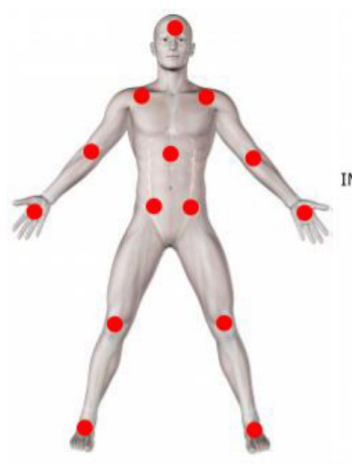

(a)

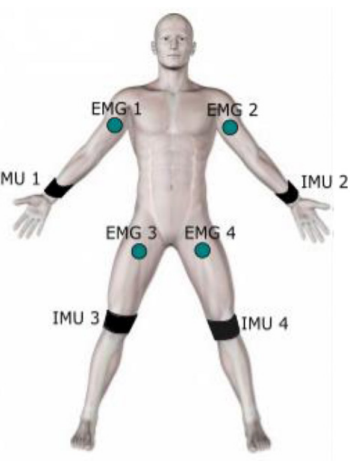

(b)
Figura 3. Distribución de sensores y punto articulados. (a) enseña los puntos articulados del Kinect. (b) distribución de los sensores IMU y EMG $m=15, k=4$. una NIUSB6211. El tamaño de ventana utilizado para definir un movimiento primitivo es 100 muestras. Se elige este tamaño, dado que en [1] se utiliza esta configuración donde resultados sugiriere un adecuado desempeño en la evaluación con este valor. $\mathrm{La}$ anterior configuración permite obtener, $20 \mathrm{sub}$ - movimientos por segundo. Luego cada ventana se codifica como $E j$ siendo $j$ es el $j$-ésimo sensor $(j \in 1 \ldots . . j)$. Posteriormente se procede a evaluar en cada una de las ventanas la transformada de Daubechies Wavalet con 35 coeficientes y 6 niveles, computando de esta manera el estimador $\boldsymbol{E} \boldsymbol{M} \boldsymbol{G F}$.

\section{ANÁLISIS DE DIMENSIONALIDAD}

Luego de encontrar el conjunto de características de los sensores se plantea analizar y determinar las características más relevantes, con el propósito de disminuir la complejidad de procesamiento, aumentar la eficiencia como la simplicidad del método. Para ello se implementa un cúmulo de técnicas de estado del arte, la cuales permiten observar y cuantificar la naturaleza de las características en baja dimensionalidad. Se replican diez técnicas de la literatura (ver Figura 4) para este análisis $[9,11,12,30,31]$. La salida de esta etapa es un matriz de característica reducida para cada uno de los sensores, así como un gráfico donde se puede realizar una lectura de que método de reducción separa adecuadamente el conjunto de características.

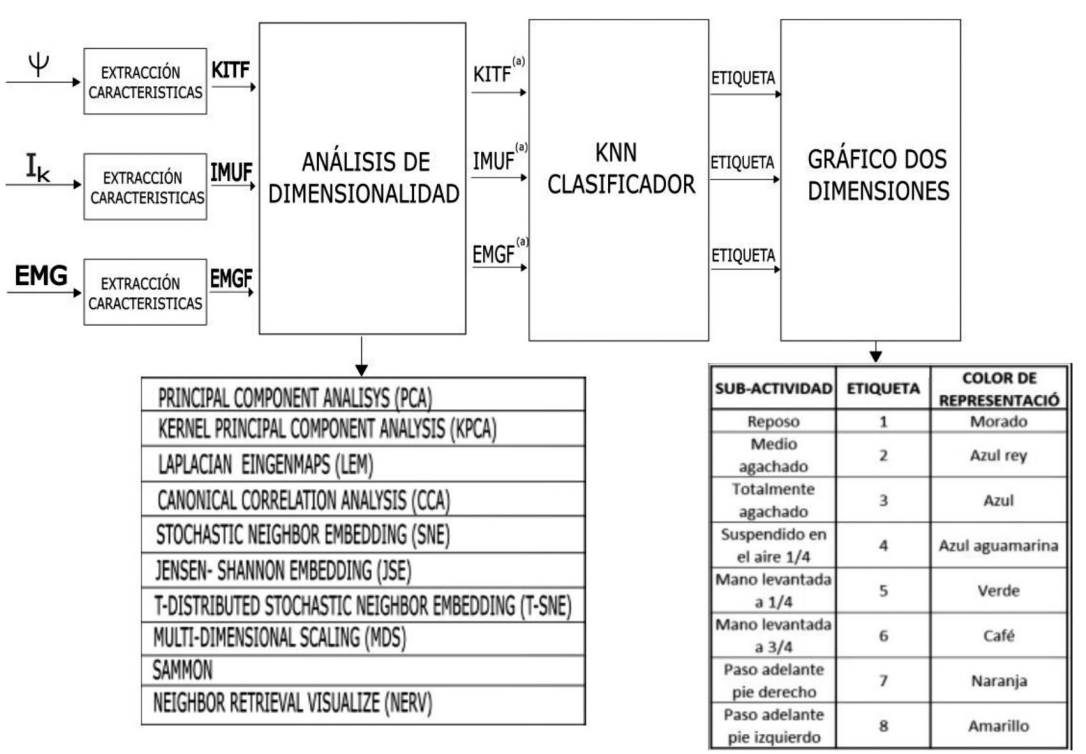

Figura 4. Procedimiento Análisis de dimensionalidad. 
Aunque el análisis de dimensionalidad permite entender la naturaleza de los datos, dos dimensiones es un criterio que puede no generar una adecuada separabilidad en la clasificación, por lo que es necesario agregar más dimensiones en el espacio de representación.

Para obtener el conjunto mínimo de características, que presente un desempeño similar al de toda la base de datos, se aplica los métodos de reducción de dimensiones enseñados en Figura 4. Esto permitirá obtener una matriz de representación reducida con la cual se debe realizar la clasificación del movimiento primitivo. Para verificar lo anterior se adopta un paradigma de validación cruzada (70\% entrenamiento y $30 \%$ prueba) aplicando el clasificador KNN. Este método permitirá calcular la eficiencia en la detección en función del número de características.

La prueba de validación consiste en la variación del número de características versus la eficiencia en obtenida en la tarea clasificación, lo anterior se realiza para $l$ repeticiones. Así el criterio busca el número de características que estabiliza la eficiencia en la etapa de reconocimiento de movimientos primitivos.

\section{RECONOCIMIENTO DE MOVIMIENTOS PRIMITIVOS}

Dado el conjunto de características reducidas $(\operatorname{KITF}(a), \operatorname{IMUF}(a), \operatorname{EMGF}(a))$ se procede con la clasificación de los movimientos primitivos siguiendo los esquemas adoptados en [1,2,3] (ver Figura 5), donde se propone adoptar un paradigma de aprendizaje supervisado por medio de máquina de soporte vectorial (SVM) en modalidad "All-pairs". La utilización de una SVM como modelo separación obedece principalmente a su robustez contra datos con ruido, método ampliamente difundido, relativamente sencillo en la implementación [1]. Es de aclarar que la ejecución de actividades físicas consta de una secuencia de acciones que se pueden dividir en ventanas de tiempo, formando así los movimientos primitivos, en la Figura 6 se aprecia como la actividad "Pararse-levantarse" tiene 3 movimientos primitivos, que, al ubicarlos en un ciclo, reconstruye el desplazamiento permitiendo que cada acción se puede representar mediante una secuencia de movimientos, los cuales generan un código único que permite obtener un factor discriminante en la clasificación de la actividad.

\section{RECONOCIMIENTO DE LA ACTIVIDAD}

Esta etapa consiste en la clasificación de las actividades definidas en Figura 1. Primeramente, se utiliza un módulo de fusión de datos que se encarga de centralizar la información entregada por la SVM, luego se reconoce la tarea aplicando

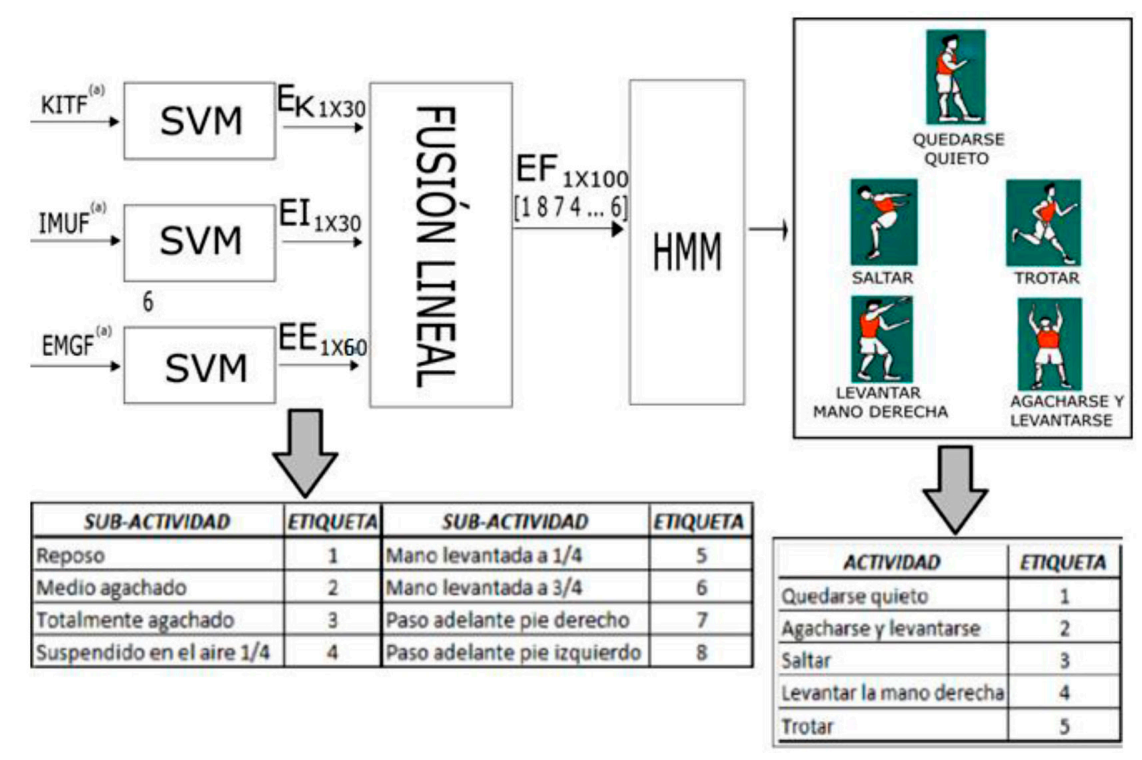

Figura 5. Instrumento de clasificación de movimientos primitivos y actividades. 


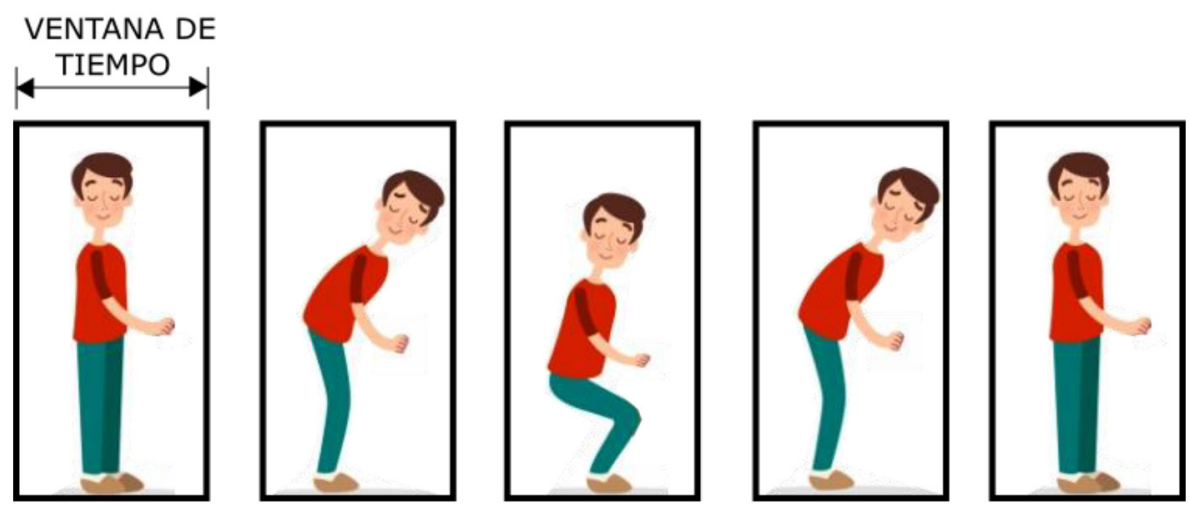

Figura 6. Construcción de Movimiento primitivo

un clasificador HMM (Hidden Markov Model), en particular se adopta este modelo a razón de que es ampliamente aplicado para análisis de secuencias $[1,4,6]$. El diagrama de bloques de este proceso se enseña en Figura 5.

\section{Fusión de datos por medio de etiquetas}

La SVM genera una etiqueta de subactividades con información provista de la red de sensores IMU, KINECT y EMG. Estos sensores proveen información del movimiento a diferentes velocidades de muestreo, así póstumo al clasificador HMM es necesario fusionar las etiquetas, este proceso consiste en generar un vector de características $E F$, este se constituye al concatenar linealmente cada etiqueta entregada por las SVM durante la ventana de observación (ver ecuación 4).

$$
E F_{1 x 120}=\left[E K_{1 \times 30}, E I_{1 \times 30}, E E_{1 \times 60}\right]
$$

Es de aclarar que la base de datos define para el sensor Kinect que un movimiento primitivo está constituido por un conjunto de tres muestras $i=\{1,2,3\}$. Dado este agrupamiento la modalidad tiene capacidad de entregar 10 movimientos primitivos por segundo, de manera que el total de sub-movimientos es de 30. Para la red IMUs también se utiliza un conjunto de 3 muestras, dando así el mismo periodo de muestro de la modalidad anterior. Para los sensores EMG cada una de las señales es segmentada en ventanas de 100 muestras definiendo esta ventana como el tamaño de un movimiento primitivo. Lo anterior evidencia que esta modalidad permite obtener 20 movimientos primitivos por segundo obteniendo 60 subactividades en total.

\section{Métricas de presentación de resultados}

Se presentarán los resultados en dos etapas, en la primera se enseña un análisis en baja dimensionalidad con el propósito de observar la naturaleza de los datos, así como el rendimiento de los algoritmos de separación planteados en la Figura 4. Luego para continuar con el procedimiento metodológico se selecciona los métodos con mejores resultados, para ello se hace uso de la técnica de k-means, la cual sustituye en esta etapa del análisis a la máquina de vectores de soporte donde el propósito es identificar de manera rápida las técnicas de separación que mejores resultados computa en la clasificación de subactividades.

En segundo lugar, se documentan los resultados de clasificación de movimientos primitivos y actividades por medio de la matriz de confusión al correr un experimento de Monte Carlo para evaluar el desempeño del método antes diferencias en el conjunto de datos de entrenamiento y validación. Por otro se anexan los tiempos de cómputo que demandan los procesos.

\section{Proceso de entrenamiento métodos de aprendizaje}

El entrenamiento de los HMM se usa 24 estados y 32 centroides en la construcción del libro de códigos, este proceso selecciona el modelo de mejor desempeño después de realizar 100 iteraciones. Para realizar el procedimiento de evaluación del modelo entrenado se utiliza una estrategia de validación cruzada, la cual fracciona la base de datos en un $70 \%$ usada en el entrenamiento, el restante $30 \%$ para el proceso de validación. este proceso realiza iteraciones de Montecarlo con criterio de paro $\operatorname{diag} M k-1 k-1 n=1 k-1 \operatorname{diag} M n-1 \leq 0,001$ 
donde $\operatorname{diag} M k$ es la el vector generado por la diagonal de la matriz de confusión y k es la iteración promedio actual de Montecarlo [1].

Para clasificar los movimientos primitivos, se utilizaron tres máquinas de vectores de soporte multiclase que utilizan los descriptores provistos en la Tabla 2. Estos clasificadores se proponen para cada una de las modalidades de sensor utilizadas en este trabajo. Como las SVM son clasificadores binarios, se utiliza una estrategia de clasificación "All-pairs" para realizar la extensión a clasificación multiclase (lo anterior para la etapa de movimiemientos primitivos). El método fue propuesto por Hastie y Tibshirani [32] y entrena una SVM por cada par de clases, obteniendo $k(k-1) / 2$ clasificadores, donde $\mathrm{K}$ es el número de clases. Después entrenar cada modelo, se realiza una calificación binaria y se considera la clase que obtuvo más votos, como etiqueta final de clase. Este método de aprendizaje además utiliza un kernel gaussiano $k x i, x j=e-$ $\gamma x i-x j 2$ con $\gamma=1 \times 10-4$ para aumentar la separabilidad de los datos.

\section{RESULTADOS}

El análisis de metodología en primer lugar busca analizar la naturaleza de los datos una vez estos son transformados al dominio de las características KITF, $\boldsymbol{I} \boldsymbol{M U} \boldsymbol{F}, \boldsymbol{E} \boldsymbol{M} \boldsymbol{G} \boldsymbol{F}$, por medio del proceso enseñado en Figura 4, donde se realiza un procedimiento de clasificación de movimiento primitivos utilizando KNN. Lo anterior permite conocer el traslape y pertinencia del conjunto de características. Determinando rápidamente si debe buscar otras formas de caracterización. En la Figura 7 se enseña los resultados obtenidos para una combinación de sensores (IMU + Kinect), nótese como algunos métodos no logran separar adecuadamente las características originando traslape. Se puede observar de la Figura 7
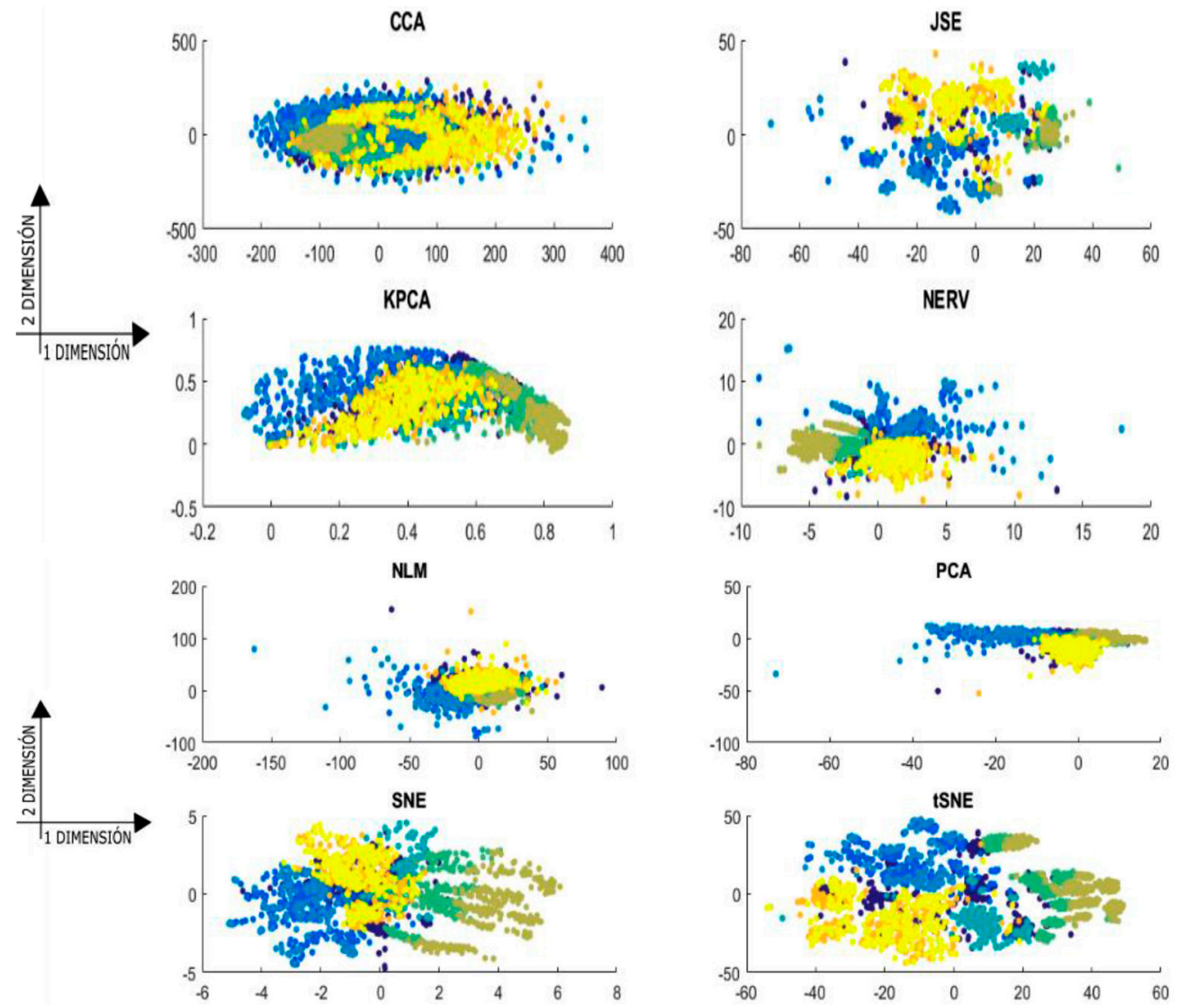

Figura 7. Análisis en 2 Dimensiones del proceso de clasificación de movimiento primitivos para los métodos de separación bajo estudio. 
que estos métodos de reducción dimensiones permiten la posibilidad de entender la naturaleza de la base datos bajos diferentes espacios de transformación. Sin embargo, se puede observar cómo los métodos JSE, SNE y tSNE logran agrupar con un mejor desempeño el espacio reducido de características asociadas al sub-movimiento. Cabe resaltar que, aunque el método [1], propone una fusión a nivel de etiquetas, en este estudio evaluamos la posibilidad de fusionar a nivel de características, para lo cual se observa la fusión Kinect + IMU y Kinect + EMG.

En Figura 8 se enseña un gráfico que asocia el rango de vecinos que se conservan, al comparar el conjunto de datos iniciales y el conjunto de datos obtenidos al aplicar los métodos de reducción de dimensionalidad. En este caso el eje de las ordenadas corresponde al porcentaje que se mantuvieron fijos, en el eje de la abscisa corresponde a la cantidad de vecinos evaluados. Para medir la tasa de vecinos que se mantienen, se calcula el área bajo la curva de esta para cada uno de los métodos bajo estudio, esta es una métrica de densidad estandarizada que toma valores entre 0,1 , es decir la relación entre los conjuntos de datos iniciales y los obtenidos luego de aplicar el método de reducción, por tanto, a mayor tasa de aceptación (cercano 1) aumenta el rendimiento del método de separación para cada una de las modalidades que se exploran. En Tabla 3 se documenta el método de menor y mayor rendimiento para algunas combinaciones de sensores.

Por otro lado, se computó el conjunto de características mínimo que permitía obtener un desempeño similar

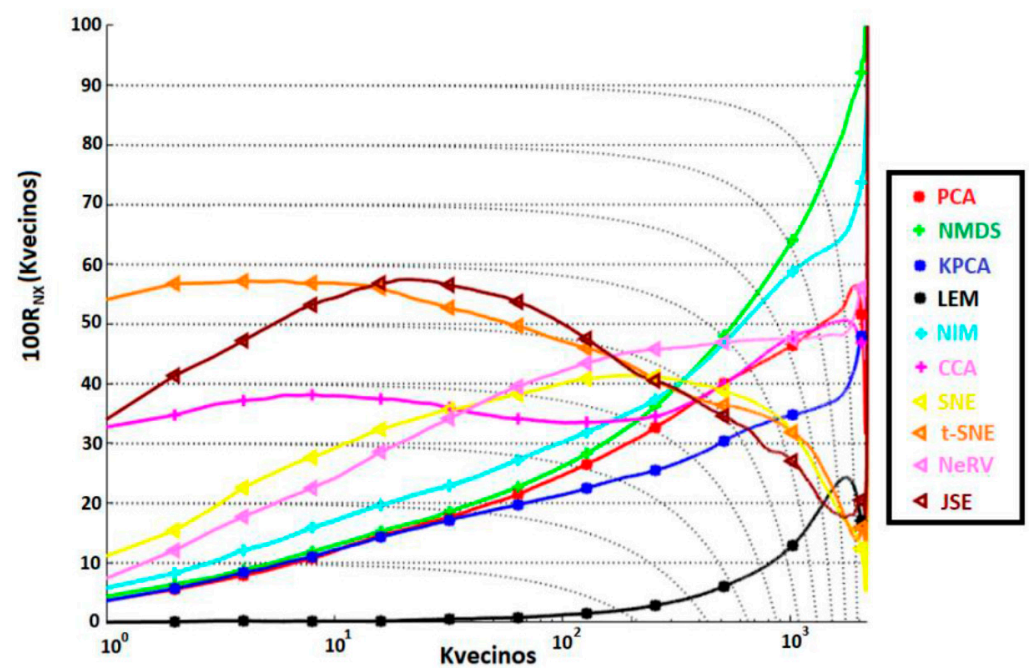

Figura 8. Probabilidad de vecinos conservados para los métodos bajo análisis.

Tabla 3. Resultados área bajo la curva Figura 8.

\begin{tabular}{|c|c|c|}
\hline Métodos & Área bajo la curva & Combinación del sensor \\
\hline tSNE & 57,4 & \multirow{2}{*}{ Sensores EMG } \\
\hline LEM & 31,3 & \\
\hline tSNE & $\begin{array}{l}50,8 \\
\end{array}$ & \multirow{2}{*}{ Sensores IMU } \\
\hline LEM & 0,7 & \\
\hline tSNE & 48,1 & \multirow{2}{*}{ Sensor Kinect } \\
\hline LEM & 2,5 & \\
\hline tSNE & 47,3 & \multirow{2}{*}{ Sensor IMU + Kinect } \\
\hline LEM & 3,3 & \\
\hline tSNE & 40,35 & \multirow{2}{*}{ Sensor EMG + Kinect } \\
\hline LEM & 29,45 & \\
\hline
\end{tabular}


al conjunto total de características en el proceso de identificación de movimientos primitivos. Para lograr lo anterior, se implementaron los métodos de reducción de características que mejor separabilidad mostraron en Figura 7 y Figura 8, luego se computó la matriz de características reducidas. Se seleccionó el criterio anterior debido a que una separabilidad mayor facilita el proceso de clasificación. Posteriormente se realizó un proceso de validación cruzada. En Tabla 4 se enseñan los resultados para los diferentes métodos propuestos. Para obtener el número de componentes necesarias, se elige el método PCA y se aplica la prueba validación nombrada anteriormente. Después de aplicar esta prueba, se determina que son necesarias 41 componentes para el descriptor $\boldsymbol{K I T F}, 30$ componentes para el descriptor $\boldsymbol{I M U} \boldsymbol{F}$, 200 componentes para el descriptor $\boldsymbol{E} \boldsymbol{M} \boldsymbol{G} \boldsymbol{F}$ y 65 componente para la combinación de características KINECT + IMU. Para la combinación KINECT + EMG no se reducen dimensiones, debido a que se requiere de todas las características para obtener un desempeño competitivo.
En la Tabla 4 se presentan los resultados de clasificación de movimientos primitivos utilizando el método K-means. Se utiliza inicialmente esta, para validar los métodos de reducción de características bajo estudio. El propósito es evaluar una estrategia de menor costo computacional para verificar la separabilidad que generan cada una de las técnicas de reducción de dimensiones para la base de datos en análisis. Del resultado de Tabla 4 se puede observar que a pesar de que el espacio de características sufre una compresión en su dimensionalidad este logra separar los sub-movimientos con desempeño similar al obtenido sin comprimir el número de características. De Tabla 4 se observa que los métodos JSE y t-SNE tienen desempeños similares y lograr acercase a la representación total de espacio, sin embargó su costo computacional es muy alto. Además, se observa que PCA es el método más adecuado para lograr la reducción, esto se debe a que logra aciertos similares al conjunto total de características y sus tiempos de ejecución son significativamente bajos con respecto a los demás

Tabla 4. Resultados del proceso de clasificación de sub actividades con y sin análisis de dimensionalidad utilizando un clasificador k-means.

\begin{tabular}{|c|c|c|c|}
\hline $\begin{array}{l}\text { Método de reducción } \\
\text { implementado }\end{array}$ & Tipo de sensor & Acierto de clasificación & $\begin{array}{l}\text { Tiempo promedio } \\
\text { de proceso }\end{array}$ \\
\hline \multirow{5}{*}{ Ninguno } & KINECT & $77,50 \pm 1,19 \%$ & $7,24[\mathrm{~s}]$ \\
\hline & IMU & $67,48 \pm 1,7 \%$ & $7,18[\mathrm{~s}]$ \\
\hline & EMG & $67,78 \pm 2,3 \%$ & $10,4[\mathrm{~s}]$ \\
\hline & KINECT+EMG & $60,50 \pm 6,25 \%$ & $11,4[\mathrm{~s}]$ \\
\hline & KINECT+IMU & $78,40 \pm 1,29 \%$ & $7,33[\mathrm{~s}]$ \\
\hline \multirow{5}{*}{ PCA } & KINECT & $76,81 \pm 1,51 \%$ & $8,98[\mathrm{~s}]$ \\
\hline & IMU & $67,54 \pm 1,99 \%$ & $7,47[\mathrm{~s}]$ \\
\hline & EMG & $60,59 \pm 2,3 \%$ & $10,41[\mathrm{~s}]$ \\
\hline & KINECT+EMG & $61,50 \pm 6,01 \%$ & $11,8[\mathrm{~s}]$ \\
\hline & KINECT+IMU & $79,63 \pm 1,98 \%$ & $7,61[\mathrm{~s}]$ \\
\hline \multirow{5}{*}{$\mathrm{t}-\mathrm{SNE}$} & KINECT & $71,44 \pm 1,31 \%$ & $655,25[\mathrm{~s}]$ \\
\hline & IMU & $64,14 \pm 1,29 \%$ & $868,77[\mathrm{~s}]$ \\
\hline & EMG & $59 \pm 2,19 \%$ & $933,47[\mathrm{~s}]$ \\
\hline & KINECT+EMG & $60,78 \pm 5,96 \%$ & $930,27[\mathrm{~s}]$ \\
\hline & KINECT+IMU & $74,24 \pm 2,13 \%$ & $890,99[\mathrm{~s}]$ \\
\hline \multirow{5}{*}{ JSE } & KINECT & $72,98 \pm 1,79 \%$ & $706,38[\mathrm{~s}]$ \\
\hline & IMU & $64,87 \pm 2,01 \%$ & $537,89[\mathrm{~s}]$ \\
\hline & EMG & $60 \pm 3,29 \%$ & $933,47[\mathrm{~s}]$ \\
\hline & KINECT+EMG & $59,89 \pm 6,79 \%$ & $993,35[\mathrm{~s}]$ \\
\hline & KINECT+IMU & $74,41 \pm 2,45 \%$ & $740,62[\mathrm{~s}]$ \\
\hline
\end{tabular}


T-SNE y JSE. Es de aclarar que las pruebas se ejecutaron en un computador ASUS- ROG G56JR, CORE i7 4700HQ (3400 MHz) y 16 GB de RAM.

Determinado el conjunto de características y los métodos adecuados para le reducción de dimensiones, se procede aplicar la reducción de dimensiones utilizando el procedimiento de Figura 5 considerando la SVM. En la Tabla 5 se enseña los resultados del clasificador de movimiento primitivos sin considerar reducción de características mientras que en la Tabla 6 con reducción de características.

De la Tabla 6 se evidencia que el método con menor desempeño es el T-SNE logrando en promedio aciertos de 69,41 \pm 0,96 para el sensor Kinect, $66,09 \pm 0,59$ para el sensor IMU y 44,32 $\pm 0,32$ para el sensor EMG. Por otro lado, los métodos JSE y PCA logran desempeños deseados y cercanos al conjunto de datos sin comprensión en las dimensiones tal y como se enseña en la Tabla 7. Estos valores varían entre el $59 \%$ y el 78,40\% acercándose a los obtenidos en la Tabla 5. Lo anterior hace que estos métodos sean candidatos para clasificar submovimientos, sin embargo, se debe tener en cuenta su costo computacional, el cual como sugiere la Tabla 4 es elevado respecto PCA.

Finalmente se procede a probar el impacto de esta reducción de dimensiones en la detección de actividades físicas, la cual se logra con la fusión de etiquetas provistas por la SVM y la clasificación con HMM. Cabe resaltar que la reducción de dimensiones se realiza a la matriz de características con lo que se entrenan las SVM y no las etiquetas con las que se entrena la HMM. El objetivo de esta

Tabla 5. Resultados del proceso de clasificación de movimientos primitivos con SVM con todo el conjunto de características.

\begin{tabular}{|c|c|c|c|}
\hline \multirow{2}{*}{ Etiqueta } & \multicolumn{3}{|c|}{ SIN REDUCCIÓN } \\
\cline { 2 - 4 } & KINECT & IMU & EMG \\
\hline E1 & $86,77 \pm 3,79$ & $86,77 \pm 0,73$ & $72,28 \pm 5,83$ \\
\hline E2 & $77,57 \pm 3,81$ & $78,71 \pm 2,10$ & $66,51 \pm 1,13$ \\
\hline E3 & $71,86 \pm 3,86$ & $71,46 \pm 4,33$ & $70,84 \pm 4,73$ \\
\hline E4 & $89,95 \pm 1,46$ & $74,52 \pm 0,93$ & $71,49 \pm 1,33$ \\
\hline E5 & $90,47 \pm 3,15$ & $76,51 \pm 5,25$ & $59,43 \pm 5,21$ \\
\hline E6 & $96,78 \pm 1,33$ & $93,07 \pm 2,02$ & $63,88 \pm 2,35$ \\
\hline E7 & $75,68 \pm 1,95$ & $61,43 \pm 2,91$ & $50,54 \pm 1,84$ \\
\hline E8 & $75,63 \pm 2,97$ & $57,56 \pm 2,73$ & $34,08 \pm 2,50$ \\
\hline Total & $84,69 \pm 3,31$ & $75,00 \pm 2,63$ & $61,13 \pm 3,12$ \\
\hline
\end{tabular}

Tabla 6. Resultados del proceso de clasificación de movimientos primitivos con SVM para los métodos T-SNE y JSE.

\begin{tabular}{|c|c|c|c|c|c|c|}
\hline \multirow{2}{*}{ Etiqueta } & \multicolumn{3}{|c|}{ T-SNE } & \multicolumn{3}{c|}{ JSE } \\
\cline { 2 - 7 } & KINECT & IMU & EMG & KINECT & IMU & EMG \\
\hline E1 & $64,36 \pm 6,07$ & $70,37 \pm 1,00$ & $29,74 \pm 2,53$ & $68,75 \pm 2,68$ & $81,97 \pm 0,03$ & $36,55 \pm 1,63$ \\
\hline E2 & $58,13 \pm 1,38$ & $60,61 \pm 1,07$ & $56,72 \pm 1,96$ & $62,05 \pm 1,16$ & $69,57 \pm 2,02$ & $59,19 \pm 3,49$ \\
\hline E3 & $66,09 \pm 4,43$ & $58,85 \pm 5,43$ & $45,32 \pm 3,55$ & $71,91 \pm 1,00$ & $65,00 \pm 5,00$ & $45,75 \pm 1,31$ \\
\hline E4 & $66,35 \pm 0,81$ & $71,52 \pm 0,70$ & $62,18 \pm 0,35$ & $78,02 \pm 2,22$ & $70,85 \pm 0,73$ & $64,68 \pm 0,55$ \\
\hline E5 & $77,99 \pm 3,11$ & $68,35 \pm 4,02$ & $36,36 \pm 2,04$ & $86,75 \pm 1,86$ & $79,46 \pm 4,46$ & $42,08 \pm 4,84$ \\
\hline E6 & $92,73 \pm 1,71$ & $85,68 \pm 2,99$ & $45,40 \pm 2,23$ & $93,42 \pm 0,95$ & $90,54 \pm 0,61$ & $49,53 \pm 3,43$ \\
\hline E7 & $67,37 \pm 2,89$ & $58,80 \pm 0,62$ & $41,32 \pm 2,68$ & $69,95 \pm 1,86$ & $55,17 \pm 0,52$ & $40,99 \pm 2,34$ \\
\hline E8 & $62,25 \pm 2,61$ & $54,58 \pm 1,88$ & $37,52 \pm 2,58$ & $60,49 \pm 3,35$ & $53,79 \pm 1,21$ & $42,24 \pm 0,18$ \\
\hline Promedio & $69,41 \pm 0,96$ & $66,09 \pm 0,59$ & $44,32 \pm 0,32$ & $73,92 \pm 0,23$ & $70,80 \pm 0,78$ & $47,63 \pm 1,44$ \\
\hline
\end{tabular}


Tabla 7. Resultados del proceso de clasificación de movimientos primitivos con SVM para los métodos SNE y PCA.

\begin{tabular}{|c|c|c|c|c|c|c|}
\hline \multirow{2}{*}{ Etiqueta } & \multicolumn{3}{|c|}{ SNE } & \multicolumn{3}{c|}{ PCA } \\
\cline { 2 - 7 } & KIINECT & IMU & EMG & KIINECT & IMU & EMG \\
\hline E1 & $70,33 \pm 6,99$ & $81,37 \pm 1,33$ & $38,73 \pm 5,40$ & $66,21 \pm 6,34$ & $82,04 \pm 1,26$ & $74,18 \pm 6,84$ \\
\hline E2 & $63,89 \pm 1,28$ & $71,57 \pm 2,34$ & $59,38 \pm 1,51$ & $64,33 \pm 2,83$ & $72,03 \pm 2,72$ & $64,45 \pm 2,27$ \\
\hline E3 & $62,87 \pm 3,86$ & $63,46 \pm 5,55$ & $49,77 \pm 4,10$ & $68,40 \pm 5,88$ & $64,84 \pm 6,44$ & $65,21 \pm 6,62$ \\
\hline E4 & $76,98 \pm 1,35$ & $74,01 \pm 2,11$ & $64,00 \pm 0,98$ & $72,40 \pm 1,18$ & $65,94 \pm 2,71$ & $60,08 \pm 0,94$ \\
\hline E5 & $86,75 \pm 3,27$ & $72,12 \pm 3,06$ & $42,94 \pm 3,09$ & $89,65 \pm 2,70$ & $72,50 \pm 5,68$ & $58,53 \pm 5,47$ \\
\hline E6 & $95,22 \pm 1,54$ & $90,63 \pm 1,50$ & $49,05 \pm 2,86$ & $95,44 \pm 1,40$ & $91,21 \pm 1,97$ & $61,98 \pm 2,52$ \\
\hline E7 & $66,06 \pm 2,59$ & $56,00 \pm 2,93$ & $43,73 \pm 1,49$ & $56,38 \pm 2,73$ & $57,31 \pm 3,16$ & $53,38 \pm 2,66$ \\
\hline E8 & $64,71 \pm 3,55$ & $52,96 \pm 3,78$ & $40,08 \pm 2,05$ & $65,45 \pm 2,85$ & $52,90 \pm 3,47$ & $53,11 \pm 3,10$ \\
\hline Total & $73,36 \pm 1,21$ & $70,27 \pm 1,56$ & $48,462 \pm 1,2$ & $72,28 \pm 1,47$ & $69,84 \pm 1,63$ & $61,36 \pm 1,71$ \\
\hline
\end{tabular}

prueba es probar que la reducción de dimensiones no interfiere con el desempeño que logra la detección de la actividad.

En Tabla 9 se enseñan los resultados de clasificación de actividades con los métodos JSE y PCA. Se aclara que la selección de estos dos métodos de reducción es debido a que fueron los que mejor desempeño presentan según en las pruebas documentadas en la Tabla 4. En Tabla 9 se evidencia que estos dos métodos presentan desempeños comparables con los obtenidos con todo el conjunto de características, ver Tabla 8. Aunque estos métodos permiten reducir el conjunto de características, el método JSE tiene un alto costo computacional, dado que, el cómputo requiere una cantidad significativa de operaciones,

Tabla 8. Resultados del proceso de clasificación de actividades con HMM con todo el conjunto de características.

\begin{tabular}{|c|c|c|c|c|}
\hline \multirow{2}{*}{ Etiqueta } & \multicolumn{4}{|c|}{ Sin Reducción } \\
\cline { 2 - 5 } & KINECT & IMU & EMG & KINECT + IMU + EMG \\
\hline E1 & $93,50 \pm 10,77$ & $99,80 \pm 1,07$ & $38,78 \pm 16,33$ & $100 \pm 0,0$ \\
\hline E2 & $90,30 \pm 9,06$ & $98,33 \pm 8,64$ & $90,94 \pm 11,23$ & $99,60 \pm 1,53$ \\
\hline E3 & $84,96 \pm 11,31$ & $96,14 \pm 15,76$ & $96,16 \pm 4,63$ & $99,62 \pm 1,03$ \\
\hline E4 & $97,16 \pm 7,39$ & $98,43 \pm 0,00$ & $94,08 \pm 3,94$ & $99,09 \pm 1,59$ \\
\hline E5 & $94,86 \pm 7,90$ & $100,00 \pm 0,00$ & $89,69 \pm 11,07$ & $95,76 \pm 3,36$ \\
\hline Total & $92,15 \pm 9,29$ & $98,18 \pm 3,22$ & $81,93 \pm 8,52$ & $98,81 \pm 1,81$ \\
\hline
\end{tabular}

Tabla 9. Resultados del proceso de clasificación de actividades con HMM para los métodos JSE y PCA.

\begin{tabular}{|c|c|c|c|c|c|c|c|c|}
\hline \multirow[b]{2}{*}{ Etiqueta } & \multicolumn{4}{|l|}{ JSE } & \multicolumn{4}{|l|}{ PCA } \\
\hline & KINECT & IMU & EMG & $\begin{array}{l}\text { KINECT + } \\
\text { IMU + EMG }\end{array}$ & KINECT & IMU & EMG & $\begin{array}{l}\text { KINECT + } \\
\text { IMU + EMG }\end{array}$ \\
\hline E1 & $95,31 \pm 8,69$ & $99,75 \pm 1,30$ & $50,93 \pm 14,87$ & $79,41 \pm 11,47$ & $89,38 \pm 8,79$ & $100,00 \pm 0,0$ & $47,49 \pm 14,1$ & $82,76 \pm 14,8$ \\
\hline E2 & $89,71 \pm 9,69$ & $93,03 \pm 8,64$ & $62,16 \pm 11,31$ & $91,16 \pm 6,59$ & $81,90 \pm 14,2$ & $92,83 \pm 8,45$ & $53,61 \pm 12,7$ & $85,26 \pm 11,2$ \\
\hline E3 & $75,45 \pm 5,89$ & $78,11 \pm 15,76$ & $76,86 \pm 12,48$ & $100,00 \pm 0,00$ & $60,74 \pm 20,8$ & $78,86 \pm 16,8$ & $51,08 \pm 12,9$ & $94,30 \pm 8,00$ \\
\hline E4 & $94,13 \pm 0,42$ & $100,00 \pm 0,00$ & $66,76 \pm 15,29$ & $92,51 \pm 8,05$ & $98,93 \pm 4,48$ & $100,00 \pm 0,0$ & $73,64 \pm 13,9$ & $99,07 \pm 3,23$ \\
\hline E5 & $78,10 \pm 6,23$ & $74,81 \pm 18,83$ & $72,44 \pm 12,54$ & $96,75 \pm 3,31$ & $74,97 \pm 19,9$ & $81,87 \pm 16,4$ & $66,99 \pm 11,8$ & $96,28 \pm 7,04$ \\
\hline Total & $86,54 \pm 5,40$ & $89,14 \pm 3,53$ & $65,83 \pm 4,52$ & $91,97 \pm 2,03$ & $81,19 \pm 5,08$ & $90,71 \pm 3,78$ & $58,56 \pm 5,49$ & $91,53 \pm 4,88$ \\
\hline
\end{tabular}


sin embargo, el método PCA realiza el trabajo con una eficiencia adecuada y el tiempo de ejecución fue dramáticamente menor en todas las pruebas.

Lo anterior se puede verificar en Tabla 10, en esta se enseña los tiempos de computo del algoritmo para diferentes modalidades de sensores y métodos de reducción de características, nótese que el método PCA presenta un menor costo computacional para todas las modalidades de sensores, caso contrario ocurre con los métodos SNE y JSE donde para todas las modalidades los recursos computacionales elevados.

\section{AGRADECIMIENTOS}

Los autores de este trabajo agradecen a la Universidad Tecnológica de Pereira y al Tecnoparque Nodo Pereira por el apoyo y uso de infraestructura en el desarrollo de la investigación.

\section{CONCLUSIONES}

En este trabajo se implementó una metodología de visualización de métodos de reducción de dimensionalidad para el reconocimiento de actividades físicas humanas. Lo anterior permite determinar el comportamiento de los datos recopilados en el trabajo [1], resaltando el aporte que genera las diferentes modalidades de sensor en la clasificación de una actividad física.

En este trabajo se estudia la contribución de cada sensor en la tarea de reconocimiento, lo anterior se logra al visualizar y validar el rendimiento de cada conjunto de características provenientes de las diferentes modalidades de sensores. Para realizar un reconocimiento de actividad robusto, es necesario usar todos los sensores debido a los posibles fallos que estos dispositivos pueden presentar durante el proceso de ejecución. Aunque se requiere la información de todos los sensores, en este trabajo se prueba que ciertas características aportan poca información para la identificación de sub movimientos, determinando que con 41 componentes para el descriptor $\boldsymbol{K I T F}$, 30 componentes para el descriptor $\boldsymbol{I M U} \boldsymbol{F}, 200$ componentes para el descriptor $\boldsymbol{E} \boldsymbol{M} \boldsymbol{G} \boldsymbol{F}$ y 65 componente para KINECT + IMU, se logran resultados similares al reportado en [1] utilizando las metodologías de reducción de dimensiones como PCA, T-SNE, SNE y JSE. Lo anterior para el caso de PCA conlleva a una reducción del costo

Tabla 10. Tiempo de cómputo para clasificar un movimiento primitivo con SVM.

\begin{tabular}{|c|c|c|c|c|}
\hline \multicolumn{5}{|c|}{ Modalidad Kinect } \\
\hline Método & Reducción [s] & Entrenamiento [s] & Clasificación [s] & Tiempo Total [s] \\
\hline Sin Reducción & No aplica & $34,515 \pm 0,98$ & $0,203 \pm 0,015$ & $34,718 \pm 0,98$ \\
\hline PCA & $4,355 \pm 0,91$ & $16,33 \pm 0,943$ & $0,00118 \pm 0,00919$ & $20,696 \pm 0,97$ \\
\hline SNE & $921,29 \pm 20,33$ & $15,83 \pm 0,934$ & $0,00138 \pm 0,00857$ & $940,13 \pm 20,3$ \\
\hline JSE & $1133,94 \pm 35,34$ & $16,43 \pm 0,915$ & $0,00109 \pm 0,00981$ & $1150,38 \pm 35,34$ \\
\hline \multirow{5}{|c|}{ Modalidad IMU } \\
\cline { 2 - 6 } & Reducción [s] & Entrenamiento [s] & Clasificación [s] & Tiempo Total [s] \\
\hline Sin Reducción & No aplica & $39,46 \pm 0,931$ & $0,301 \pm 0,015$ & $39,761 \pm 1,79$ \\
\hline PCA & $6,423 \pm 1,21$ & $30,33 \pm 0,896$ & $0,00091 \pm 0,0012$ & $36,753 \pm 1,53$ \\
\hline SNE & $842,22 \pm 19,37$ & $29,47 \pm 0,887$ & $0,00098 \pm 0,0013$ & $873,44 \pm 22,21$ \\
\hline JSE & $947,24 \pm 25,79$ & $31,11 \pm 0,891$ & $0,00092 \pm 0,0011$ & $978,36 \pm 26,79$ \\
\hline & \multicolumn{5}{|c|}{ Modalidad EMG } \\
\cline { 2 - 6 } & Reducción [s] & Entrenamiento [s] & Clasificación [s] & Tiempo Total [s] \\
\hline Sin Reducción & No aplica & $41,46 \pm 1,38$ & $0,341 \pm 0,0168$ & $41,801 \pm 2,38$ \\
\hline PCA & $8,743 \pm 1,78$ & $41,27 \pm 1,69$ & $0,00101 \pm 0,0039$ & $50,01 \pm 2,89$ \\
\hline SNE & $1372,43 \pm 38,47$ & $40,91 \pm 1,93$ & $0,00103 \pm 0,0037$ & $1413,35 \pm 44,38$ \\
\hline JSE & $1610,04 \pm 45,78$ & $41,32 \pm 1,79$ & $0,0010 \pm 0,0041$ & $1660,37 \pm 48,36$ \\
\hline
\end{tabular}


computacional y hace que metodologías robustas como [1] puedan tener un menor costo temporal a la hora de su implementación. Lo anterior puede ser verificado con la información de las Tabla 8 , Tabla 9 y Tabla 10.

Con la inclusión de diferentes procedimientos utilizados en la investigación, se pudo reducir el conjunto de características utilizadas a una representación adecuada tal que al hacer uso de un clasificador supervisado se tiene un porcentaje de acierto sobresaliente y que puede servir como elemento de partida en la implementación de un entorno de seguimiento y control con los sistemas multimodales. Lo resultados sugieren que PCA es el método más adecuado para obtener un espacio de representación reducido, esto se debe a que logra aciertos similares al conjunto total de características y sus tiempos de ejecución son significativamente bajos con respecto a las metodologías del estado del arte como T-SNE, SNE y JSE.

Este método también podría ser aplicado a otros problemas similares, debido a que son útiles para analizar la naturaleza de los datos, además de reducir el conjunto de características. Siendo así una herramienta importante para la selección de características relevantes en tareas de clasificación.

\section{REFERENCIAS}

[1] A.F. Calvo, G. A. Holguín and H. Medeiros. "Human activity recognition using multimodal data fusion". 23rd Iberoamerican Congress, CIARP Iberoamerican Congress on Pattern Recognition. Madrid, Spain. 2018.

[2] T. Helten, M. Müller, H.P. Seidel and C. Theobalt. "Real-time body tracking with one depth camera and inertial sensors". 2013 IEEE International Conference Computer Vision (ICCV). Sydney, Australia. 2013.

[3] D.M. De Castro. "Aplicación Android para el reconocimiento automático de actividades físicas en tiempo real". Master's thesis, Universidad Carlos III de Madrid Departamento de Informática. Fecha de consulta: 4 de febrero de 2019.URL: https://earchivo.uc3m.es/hand le/10016/17138

[4] M. Zhang and A.A. Sawchuk. "Motion primitive-based human activity recognition using a bag-of-features approach”. ACM
SIGHIT International Health Informatics Symposium (IHI). New York, USA. 2012.

[5] F. Destelle, A. Ahmadi, N. E. O'Connor, K. Moran, A. Chatzitofis, D. Zarpalas and P. Daras. "Low-cost accurate skeleton tracking based on fusion of kinect and wearable inertial sensors". 22nd European Signal Processing Conference (EUSIPCO). Lisbon, Portugal. 2014.

[6] Z. Zhang, Y. Liu, A. Li and M. Wang. "A novel method for user-defined human posture recognition using Kinect". 7th International Congress on Image and Signal Processing (CISP). Dalian, China. 2014.

[7] D. Rengifo y J.F. Gallego. "Comparación de técnicas de reducción de dimensionalidad para la clasificación de actividades físicas humanas utilizando métodos estadísticos". Trabajo de grado ingeniería electrónica. Universidad Tecnológica de Pereira. Fecha de consulta: 5 de marzo de 2019. URL: http://repositorio.utp.edu.co/dspace/ bitstream/hand le/11059/7288/6213678G166. pdf?sequence $=1$

[8] H, Wu. W. Pan, X. Xiong and S. Xu: "Human activity recognition based on the combined SVM \& HMM". 2014 IEEE International Conference on Information and Automation (ICIA). Hailar, China. 2014.

[9] L. De Lathauwer, L.B. De Moor and J. Vandewalle. "Dimensionality reduction in higher-order-only ICA". 68th IEEE Signal Processing Workshop on Higher-Order Statistics. Alberta, Canada. 1997.

[10] S. Mishra, D. Mishra, S. Das and A.K. Rath. "Feature reduction using principal component analysis for agricultural data set". 3rd International Conference on Electronics Computer Technolog. Electronics Computer Technology (ICECT). Kanykumari, India. 2011.

[11] I.T. Jolliffe. "Principal component analysis". Springer series in statistics. 2nd edition, pp. 32-80. New York, USA. ISBN 978-0-387-22440-4.2002.

[12] L. V. D. Maaten and G. Hinton. "Visualizing Data using t-SNE". Journal of Machine Learning Research (JMLR). Vol. 9. No 86, pp. 2579-2606. ISNN: 2579-2605.2008.

[13] A.J. Bernheim Brush, J. Krumm and J.U. SCOTT. "Activity recognition research: 
The good, the bad and the future". 8th International Conference on Pervasive Computing. Helsinki, Finlandia. 2010.

[14] W.J. Kang, J.R. Shiu, C.K. Cheng, J.S. Lai, H.W. Tsao and T.S. Kuo. "The application of cepstral coefficients and maximum likelihood method in EMG pattern recognition". IEEE Transactionson Biomedical Engineering. Vol. $42 \mathrm{~N}^{\circ} 8$, pp. 777-785. 1995. ISNN electronico: 1558-2531. DOI: 10.1109/ 10.398638 .

[15] M.S. AL-Quraishi, A. J. Ishak, S.A. Ahmad and M.K. Hasan. "Impact of feature extraction techniques on classification accuracy for EMG based ankle joint movements". 10th Asian Control Conference (ASCC). Kota Kinabalu, Malaysia. 2015.

[16] G. Tao, P.S. Archambault and M.F. Levin. "Evaluation of Kinect skeletal tracking in a virtual reality rehabilitation system for upper limb hemiparesis". Virtual Rehabilitation (ICVR). 2013 International Conference on. Filadelfia, USA. 2013.

[17] M. Zhang and A.A. Sawchuk. "A feature selection-based framework for human activity recognition using wearable multimodal sensors". International Conference on Body Area Networks (BodyNets). Beijing, China. 2011.

[18] O. Baños, H. Pomares and I. Rojas. "Hibridación de paradigmas de clasificación en el contexto del reconocimiento automático de actividades físicas cotidianas". Congreso Español sobre Tecnologías y Lógica Fuzzy (ESTYLF). Huelva, España. 2010.

[19] F. Destelle, A. Ahmadi, N.E. O'Connor, K. Moran, A. Chatzitofis, D. Zarpalas and P. Daras. "Low-cost accurate skeleton tracking based on fusion of kinect and wearable inertial sensors". 22nd European Signal Processing Conference (EUSIPCO). Lisbon, Portugal. 2014.

[20] O.F. Ertugrul, M. E. Tagluk, Y. Kaya and R. tekin. "EMG signal classification by extreme learning machine". 21st Signal Processing and Communications Applications Conference (SIU). Haspolat, Turquía. 2013.

[21] B. Zhang and S. Zhang. "The estimation of grasping force based on the feature extracted from EMG signals". 2016 IEEE Advanced Information Management,
Communicates, Electronic and Automation Control Conference (IMCEC). Xi' an, China. 2016.

[22] N. Daisuke, Y. Wenwei, M. Masaharu, W. Ichiro, Y. Hiroshi, M. Yukio and K. Yukinori. "On-line Learning Based Electromyogram to Forearm Motion Classifier with Motor Skill Evaluation". International Journal Series. (JSME). Vol. 43 No 4, pp. 906-915. 2000. DOI: 10. 12 99/jsmec.43.906.

[23] S.-P. Lee, J.-S. Kim and S.-H. Park. "An enhanced feature extraction algorithm for EMG pattern classification". IEEE Transactions on Rehabilitation Engineering. Vol. $4 \mathrm{~N}^{\circ}$ 4, pp. 439-443. 1996. ISSN 10636528. DOI: 10.1109 / 86.547948.

[24] Z.M. Fadlullah et al. "State-of-the-art deep learning: Evolving machine intelligence toward tomorrow's intelligent network traffic control systems". IEEE Communications Surveys Tutorials. Vol. $19 \mathrm{~N}^{\circ}$ 4, pp. 2432-2455. Eectronic ISSN: 1553-877X, CD: 2372-745X. DOI: 10.1109/COMST.2017.2707140

[25] V.K. Mishra, V. Bajaj and A. Kumar. "Classification of normal, ALS, and myopathy EMG signals using ELM classifier". 2nd International Conference on Advances in Electrical, Electronics, Information, Communication and Bioinformatics (AEEICB). Chennai, India. 2016.

[26] S. Ferguson and G.R. Dunlop. "Grasp recognition from myoelectric signals". Australasian Conference on Robotics and Automation (ACRA). Auckland, Australia. 2002.

[27] I.M. Donovan, J. Puchin, K. Okada and X. Zhang. "Simple space-domain features for low-resolution EMG pattern recognition". 39th Annual International Conference of the IEEE Engineering in Medicine and Biology Society (EMBC). Seogwipo, South Korea. 2017.

[28] Z. Ju, G. Ouyang, M. Wilamowska-korsak and H. Liu. "Surface EMG based hand manipulation identification via nonlinear feature extraction and classification". IEEE Sensors Journal. Vol. $13 \mathrm{~N}^{\circ}$ 9, pp. 33023311.2013. ISSN 1530-437X. 1.1, 1.2, 2, 8.1. DOI: 10.1109/ JSEN.2013.2259051.

[29] V.A. Gutiérrez Quintero. "Definición de componentes mínimas para el reconocimiento automático de actividades físicas humanas 
utilizando una red de acelerómetros". Tesis pregrado ingeniería electrónica. Facultad de ingeniería Universidad Tecnológica de Pereira. Fecha de consulta: 6 de febrero de 2019. URL: http://repositorio.utp.edu.co/ dspace/ handle/11059/6350

[30] L.J. Cao, K.S. Chua, W.K. Chong, H.P. Lee and Q.M. Gu. "A comparison of PCA, KPCA and ICA for dimensionality reduction in support vector machine". Neurocomputing. Vol. $55 \mathrm{~N}^{\circ} 1-2$, pp. 321-336. 2003. DOI: 10.1016 / S0925-2312.
[31] J. Venna, J. Peltonen, K. Nybo, H. Aidos and S. Kask. "Information Retrieval Perspective to Nonlinear Dimensionality Reduction for Data Visualization”. Journal of Machine Learning Research (JMLR). Vol. 11, pp. 451490.2010. DOI: 10.1145/1756006.1756019.

[32] G.H. Halldorsson, J.A. Benediktsson and J.R. Sveinsson. "Support Vector Machines in Multisource Classification". 2003 IEEE International Geoscience and Remote Sensing Symposium (IGARSS). Vol. 3. Toulouse, Francia. 2003. 